\title{
Expanding repellers in limit sets for iterations of holomorphic functions
}

by

\author{
Feliks Przytycki (Warszawa)
}

\begin{abstract}
We prove that for $\Omega$ being an immediate basin of attraction to an attracting fixed point for a rational mapping of the Riemann sphere, and for an ergodic invariant measure $\mu$ on the boundary $\operatorname{Fr} \Omega$, with positive Lyapunov exponent, there is an invariant subset of Fr $\Omega$ which is an expanding repeller of Hausdorff dimension arbitrarily close to the Hausdorff dimension of $\mu$. We also prove generalizations and a geometric coding tree abstract version. The paper is a continuation of a paper in Fund. Math. 145 (1994) by the author and Anna Zdunik, where the density of periodic orbits in Fr $\Omega$ was proved.
\end{abstract}

1. Introduction. Let $\Omega$ be a simply connected domain in $\overline{\mathbb{C}}$ and $f$ be a holomorphic map defined on a neighbourhood $W$ of $\operatorname{Fr} \Omega$ to $\overline{\mathbb{C}}$. Assume $f(W \cap \Omega) \subset \Omega, f(\operatorname{Fr} \Omega) \subset \operatorname{Fr} \Omega$ and $\operatorname{Fr} \Omega$ repells to the side of $\Omega$, that is, $\bigcap_{n=0}^{\infty} f^{-n}(W \cap \bar{\Omega})=\operatorname{Fr} \Omega$. An important special case is where $\Omega$ is an immediate basin of attraction of an attracting fixed point for a rational function. This covers also the case of a component of the immediate basin of attraction to a periodic attracting orbit, as one can consider an iterate of $f$ mapping the component to itself. Distances and derivatives are considered in the Riemann spherical metric on $\overline{\mathbb{C}}$.

Let $R: \mathbb{D} \rightarrow \Omega$ be a Riemann mapping from the unit disc onto $\Omega$ and let $g$ be a holomorphic extension of $R^{-1} \circ f \circ R$ to a neighbourhood of the unit circle $\partial \mathbb{D}$. It exists and it is expanding on $\partial \mathbb{D}$ (see [P2, Section 7]). We prove the following

TheOREM A. Let $\nu$ be an ergodic g-invariant probability measure on $\partial \mathbb{D}$ such that for $\nu$-a.e. $\zeta \in \partial \mathbb{D}$ the radial limit $\widehat{R}(\zeta):=\lim _{r}{ }_{1} R(r \zeta)$ exists. Assume that the measure $\mu:=\widehat{R}_{*}(\nu)$ has positive Lyapunov exponent $\chi_{\mu}(f)$.

2000 Mathematics Subject Classification: Primary 37F15; Secondary 37F35, 37D25.

Key words and phrases: boundary of basin of attraction, iteration of rational map, Hausdorff dimension, hyperbolic dimension, coding tree, Pesin theory, Katok theory.

Supported by Polish KBN grant 2P03A 03425. 
Let $\varphi: \partial \mathbb{D} \rightarrow \mathbb{R}$ be a continuous real-valued function. Then for every $\varepsilon>0$ there exist a g-invariant expanding repeller $Y \subset \partial \mathbb{D}$ and $C>0$ such that for all positive integers $n$ and all $\zeta \in Y$,

(i) $-\ln C+n\left(\int \varphi d \nu-\varepsilon\right) \leq \sum_{j=0}^{n-1} \varphi\left(g^{j}(\zeta)\right) \leq \ln C+n\left(\int \varphi d \nu+\varepsilon\right)$.

(ii) $\widehat{R}$ is defined on all of $Y$ and finite-to-one on $Y$. Moreover $R(r \zeta) \rightarrow$ $\widehat{R}(\zeta)$ uniformly as $r \nearrow 1$ for $\zeta \in Y$. The set $X:=\widehat{R}(Y)$ is an expanding repeller for $f$ contained in $\operatorname{Fr} \Omega$. Both $Y$ and $X$ are Cantor sets.

(iii) $C^{-1} \exp n\left(\chi_{\mu}(f)-\varepsilon\right) \leq\left|\left(f^{n}\right)^{\prime}(\widehat{R}(\zeta))\right| \leq C \exp n\left(\chi_{\mu}(f)+\varepsilon\right)$.

(iv) $\operatorname{HD}(X) \geq \operatorname{HD}(\mu)-\varepsilon$.

The existence of an expanding repeller $X \subset \operatorname{Fr} \Omega$ satisfying (iii) for all $x \in X$ (in place of $\widehat{R}(\zeta)$ ) and (iv) holds without the assumption that $\Omega$ is simply connected.

Above, $X$ being an expanding repeller for $f$ means that $X$ is compact, $f(X) \subset X$ and the map $f$ restricted to $X$ is open, topologically mixing and expanding, that is, there exist $C>0$ and $\lambda>1$, called an expanding constant, such that $\left|\left(f^{n}\right)^{\prime}(x)\right| \geq C \lambda^{n}$ for every $x \in X$. The property that $\left.f\right|_{X}$ is open is equivalent to the existence of a neighbourhood $U$ of $X$ in $\mathbb{C}$, called a repelling neighbourhood, such that every forward $f$-trajectory $x, f(x), \ldots, f^{n}(x), \ldots$ staying in $U$ must be contained in $X$. The definition of an expanding repeller $Y \subset \partial \mathbb{D}$ for $g$ is similar. $\operatorname{HD}(X)$ denotes the Hausdorff dimension of the set $X$, and $\operatorname{HD}(\mu)$ the Hausdorff dimension of the measure $\mu$ which is defined as the infimum of the Hausdorff dimensions of sets of full measure $\mu$.

Property (iv) is a version of the fact that the hyperbolic Hausdorff dimension of the Julia set $J(f)$ for a rational mapping (= supremum of the Hausdorff dimensions of expanding repellers contained in $J(f))$ is equal to the hyperbolic dynamical dimension (= supremum of the Hausdorff dimensions of invariant probability measures on $J(f)$ of positive Lyapunov exponents); see for example [PU].

Theorem A, with property $\left(\mathrm{v}^{\prime}\right)$ below added to the conclusions, extends the main theorem from the paper [PZ], where the density of periodic orbits in $\operatorname{Fr} \Omega$ was proved. The idea of the proof, as in [PZ], is to apply Pesin and Katok theories; see [HK, Suplement] for a general theory and [PU, Ch. 9] for its adaptation in holomorphic iteration. The problem is, as in [PZ], that the standard Katok method to produce a large hyperbolic (here expanding) set does not guarantee that the set is in $\operatorname{Fr} \Omega$. It does not give the set $Y$ either.

We needed this theorem in [P3], applied to $\varphi=\ln \left|g^{\prime}\right|$ and $\mu$ in the harmonic measure class, but it is of independent interest, so we have decided to put it in a separate paper. 
2. Additional properties. The following additional properties of suitably constructed $X$ in Theorem A will be proved:

(v) $X$ can be arbitrarily close to the topological support $\operatorname{supp} \mu$ in the Hausdorff metric in the space of compact subsets of $\operatorname{Fr} \Omega$.

(vi) For any finite families of real-valued continuous functions $\varphi_{1}, \ldots, \varphi_{k}$ on $\partial \mathbb{D}, \psi_{1}, \ldots, \psi_{k^{\prime}}$ on Fr $\Omega$, for every $i=1, \ldots, k$ and $i=1, \ldots, k^{\prime}$ respectively, for all $\zeta \in Y, x \in X$ and positive integers $n$,

$$
\begin{gathered}
-\ln C+n\left(\int_{\partial \mathbb{D}} \varphi_{i} d \nu-\varepsilon\right) \leq \sum_{j=0}^{n-1} \varphi_{i}\left(g^{j}(\zeta)\right) \leq \ln C+n\left(\int_{\partial \mathbb{D}} \varphi_{i} d \nu+\varepsilon\right), \\
-\ln C+n\left(\int_{\mathrm{Fr} \Omega} \psi_{i} d \mu-\varepsilon\right) \leq \sum_{j=0}^{n-1} \psi_{i}\left(f^{j}(x)\right) \leq \ln C+n\left(\int_{\operatorname{Fr} \Omega} \psi_{i} d \mu+\varepsilon\right) .
\end{gathered}
$$

(vii) For $\mathrm{P}$ denoting the topological pressure and $\mathrm{h}_{\text {top }}$ the topological entropy,

$$
\begin{aligned}
& \mathrm{P}\left(\left.f\right|_{X}, \psi_{i}\right) \geq \mathrm{h}_{\mu}(f)+\int_{\operatorname{Fr} \Omega} \psi_{i} d \mu-\varepsilon, \\
& \mathrm{P}\left(\left.g\right|_{Y}, \varphi_{i}\right) \geq \mathrm{h}_{\nu}(g)+\int_{\partial \mathbb{D}} \varphi_{i} d \nu-\varepsilon,
\end{aligned}
$$

in particular

(viii) $\mathrm{h}_{\text {top }}\left(\left.f\right|_{X}\right) \geq \mathrm{h}_{\mu}(f)-\varepsilon$ and $\mathrm{h}_{\text {top }}\left(\left.g\right|_{Y}\right) \geq \mathrm{h}_{\nu}(g)-\varepsilon$.

(xi) $\operatorname{HD}(Y) \geq \operatorname{HD}(\nu)-\varepsilon$.

Remark 1. Property (v) implies

$\left(\mathrm{v}^{\prime}\right)$ If $\operatorname{supp} \mu=\operatorname{Fr} \Omega$ then $X$ is arbitrarily close to $\operatorname{Fr} \Omega$ in the Hausdorff metric.

The assumption supp $\mu=\operatorname{Fr} \Omega$ holds for every $\mu=\widehat{R}_{*}(\nu)$ for $\nu$ being a $g$-invariant Gibbs state (measure) for a Hölder continuous potential function on $\partial \mathbb{D}$ (see [PZ]). In this case $\nu$ has positive entropy, hence the existence of the radial limit $\nu$-a.e. holds automatically (see $[\mathrm{PZ}]$ and references there, in particular [P1]). This automatically implies $\chi_{\mu}(f)>0$, since $0<\mathrm{h}_{\nu}(g)=$ $\mathrm{h}_{\mu}(f) \leq 2 \chi_{\mu}(f)$ (Ruelle inequality).

REMARK 2. The radial convergence in (ii) automatically implies the nontangential convergence. This means the following: For every $\zeta \in \partial \mathbb{D}$, $0<\vartheta<\pi / 2$ and $t>0$ define

$$
S_{\vartheta, t}(\zeta)=\zeta \cdot(1+\{x \in \mathbb{C} \backslash\{0\}: \pi-\vartheta \leq \operatorname{Arg}(x) \leq \pi+\vartheta,|x|<t\}) .
$$

Such a set is called a Stolz angle. If $t$ is irrelevant we skip it and write $S_{\vartheta}$. Now (ii) can be written as 
(ii') For every $0<\vartheta<\pi / 2$ the convergence $R(z) \rightarrow \widehat{R}(\zeta)$ is uniform for $\zeta \in X$ as $z \rightarrow \zeta$ and $z \in S_{\vartheta}$. The rate of convergence is exponential, more precisely, there exists $C>0$ such that for $z \in S_{\vartheta, r}(\zeta)$,

$$
\begin{aligned}
C^{-1}(1-r)^{\chi_{\mu}(f) /\left(\chi_{\nu}(g)-\varepsilon\right)} & \leq \operatorname{dist}(R(z), \widehat{R}(\zeta)) \\
& \leq C(1-r)^{\chi_{\nu}(g) /\left(\chi_{\mu}(f)+\varepsilon\right)} .
\end{aligned}
$$

3. Geometric coding tree version. As in $[\mathrm{PZ}]$, we prove a more general, abstract version of these results, in the language of a geometric coding tree. We recall the definitions and notation:

Let $U$ be an open connected subset of the Riemann sphere $\overline{\mathbb{C}}$. Consider any holomorphic mapping $f: U \rightarrow \overline{\mathbb{C}}$ such that $f(U) \supset U$ and $f: U \rightarrow f(U)$ is a proper map. Define $\operatorname{Crit}(f)=\left\{z: f^{\prime}(z)=0\right\}$, the set of critical points for $f$. Suppose that $\operatorname{Crit}(f)$ is finite. Consider any $z \in f(U)$. Let $z^{1}, \ldots, z^{d}$ be some of the $f$-preimages of $z$ in $U$ where $d \geq 2$. Consider continuous curves $\gamma^{j}:[0,1] \rightarrow f(U), j=1, \ldots, d$, joining $z$ to $z^{j}$ respectively (i.e. $\left.\gamma^{j}(0)=z, \gamma^{j}(1)=z^{j}\right)$ such that there are no critical values for the iterates of $f$ in $\bigcup_{j=1}^{d} \gamma^{j}$, i.e. $\gamma^{j} \cap f^{n}(\operatorname{Crit}(f))=\emptyset$ for every $j$ and $n>0$.

Let $\Sigma^{d}:=\{1, \ldots, d\}^{\mathbb{Z}^{+}}$denote the one-sided shift space and $\sigma$ the shift to the left, i.e. $\sigma\left(\left(\alpha_{n}\right)\right)=\left(\alpha_{n+1}\right)$. For every sequence $\alpha=\left(\alpha_{n}\right)_{n=0}^{\infty} \in \Sigma^{d}$ we define $\gamma_{0}(\alpha):=\gamma^{\alpha_{0}}$. Suppose that for some $n \geq 0$, every $0 \leq m \leq n$, and all $\alpha \in \Sigma^{d}$, the curves $\gamma_{m}(\alpha)$ are already defined. Suppose that for $1 \leq m \leq n$ we have $f \circ \gamma_{m}(\alpha)=\gamma_{m-1}(\sigma(\alpha))$, and $\gamma_{m}(\alpha)(0)=\gamma_{m-1}(\alpha)(1)$.

Define the curves $\gamma_{n+1}(\alpha)$ so that the previous equalities hold by taking suitable $f$-preimages of $\gamma_{n}$. For every $\alpha \in \Sigma^{d}$ and $n \geq 0$ set $z_{n}(\alpha):=$ $\gamma_{n}(\alpha)(1)$. Note that $z_{n}(\alpha)$ and $\gamma_{n}(\alpha)$ depend only on $\left(\alpha_{0}, \ldots, \alpha_{n}\right)$ so sometimes we consider $z_{n}$ and $\gamma_{n}$ as functions on blocks of symbols of length $n+1$. Sometimes it is convenient to denote $z$ by $z_{-1}$.

The graph $\mathcal{T}\left(z, \gamma^{1}, \ldots, \gamma^{d}\right)$ with vertices $z$ and $z_{n}(\alpha)$ and edges $\gamma_{n}(\alpha)$ is called a geometric coding tree with root at $z$. For every $\alpha \in \Sigma^{d}$ the subgraph composed of $z, z_{n}(\alpha)$ and $\gamma_{n}(\alpha)$ for all $n \geq 0$ is called a geometric branch and denoted by $b(\alpha)$.

For each $j=1, \ldots, d$ we define $f_{j}^{-1}$ on a small neighbourhood of $z$ as the branch of $f^{-1}$ mapping $z$ to $z^{j}$. For each $\alpha \in \Sigma^{d}$ the branch $f_{j}^{-1}$ has an analytic continuation $f_{j, \alpha}^{-1}$ along the curve $b(\alpha)$. Note that by construction $f_{j, \alpha}^{-1}(b(\alpha))=b(j \alpha)$, where $j \alpha$ is the concatenation of the symbol $j$ and the sequence $\alpha$. By induction, for any block $w$ of $k$ symbols in $\{1, \ldots, d\}$, for $f_{w}^{-k}$ being the branch of $f^{-k}$ mapping $z$ to $z_{k-1}(w)$ and for $f_{w, \alpha}^{-k}$ being the analytic continuation along $b(\alpha)$, we get

$$
f_{w, \alpha}^{-k}(b(\alpha))=b(w \alpha) .
$$


Similar notation is used and properties hold for finite sequences $\alpha$, where for $\alpha=\left(\alpha_{0}, \ldots, \alpha_{n}\right), b(\alpha)$ is the path in $\mathcal{T}$ from $z$ to $z_{n}(\alpha)$.

For infinite $\alpha$ the branch $b(\alpha)$ is called convergent if the sequence $\gamma_{n}(\alpha)$ is convergent to a point in $\mathrm{cl} U$ in the Hausdorff metric. We define the coding map $z_{\infty}: \mathcal{D}\left(z_{\infty}\right) \rightarrow \operatorname{cl} U$ by $z_{\infty}(\alpha):=\lim _{n \rightarrow \infty} z_{n}(\alpha)$ on the domain $\mathcal{D}=\mathcal{D}\left(z_{\infty}\right)$ of all $\alpha$ 's for which $b(\alpha)$ is convergent.

For each geometric branch $b(\alpha)$ denote by $b_{m}(\alpha)$ the part of $b(\alpha)$ starting from $z_{m}(\alpha)$, i.e. consisting of the vertices $z_{k}(\alpha), k \geq m$, and of the edges $\gamma_{k}(\alpha), k>m$.

If the map $f$ extends holomorphically to a neighbourhood of the closure of the limit set $\Lambda$ of a geometric coding tree, $\Lambda=z_{\infty}\left(\mathcal{D}\left(z_{\infty}\right)\right)$, then $\Lambda$ is called a quasi-repeller (see [PUZ]). Note that $f(\Lambda) \subset \Lambda$ and $f z_{\infty}=z_{\infty} \sigma$.

Theorem B. Let $\Lambda$ be a quasi-repeller for a geometric coding tree $\mathcal{T}\left(z, \gamma^{1}, \ldots, \gamma^{d}\right)$ for a holomorphic map $f: U \rightarrow \overline{\mathbb{C}}$. Let $\nu$ be an ergodic $\sigma$-invariant probability measure on $\Sigma^{d}$ such that for $\nu$-a.e. $\alpha \in \Sigma^{d}$ the limit $z_{\infty}(\alpha)$ exists. Assume that the measure $\mu:=z_{\infty}(\nu)$ has positive Lyapunov exponent $\chi_{\mu}(f)$. Let $\varphi, \varphi_{j}, \psi_{j}$ be continuous real-valued functions on $\Sigma^{d}$ or $\operatorname{cl} \Lambda$ respectively. Then all the properties (i)-(ix) hold, with $\widehat{R}: \partial \mathbb{D} \rightarrow \operatorname{Fr} \Omega$ replaced by $z_{\infty}: \Sigma^{d} \rightarrow \mathrm{cl} \Lambda$ defined $\nu$-a.e. and $R(r \zeta) \rightarrow \widehat{R}(\zeta)$ replaced by $\gamma_{n}(\alpha) \rightarrow z_{\infty}(\alpha)$ as $n \rightarrow \infty$.

The assumption that $z_{\infty}(\alpha)$ exists for $\nu$-a.e. $\alpha \in \Sigma^{d}$, i.e. $\nu(\mathcal{D})=1$, holds for every $\nu$ of positive entropy (compare Remark 1; see [PZ, Convergence Theorem], where further references are given). As in the Riemann mapping case, $\chi_{\mu}(f)>0$ then holds automatically.

In the setting of Theorem B property $\left(\mathrm{v}^{\prime}\right)$ also holds, with $\operatorname{Fr} \Omega$ replaced by $\mathrm{cl} \Lambda$, which immediately follows from $(\mathrm{v})$.

The assumption $\operatorname{supp} \mu=\operatorname{cl} \Lambda$ holds whenever $\nu$ is a $\sigma$-invariant Gibbs state for a Hölder continuous function on $\Sigma^{d}$ (cf. Remark 1), and if additionally the tree $\mathcal{T}$ satisfies $\gamma^{j} \cap \operatorname{cl}\left(\bigcup_{n \geq 0} f^{n}(\operatorname{Crit} f)\right)=\emptyset$ for all $j=1, \ldots, d$ and there exists a neighbourhood $U^{j} \subset f(U)$ of $\gamma^{j}$ such that area $\left(f^{-n}\left(U^{j}\right)\right) \rightarrow 0$, where area denotes the standard Riemann measure on $\overline{\mathbb{C}}$.

For the proof see [PZ, Lemma 3], where $\operatorname{cl} \Lambda$ is replaced by a formally larger set $\widehat{\Lambda}:=\left\{\right.$ all limit points of the sequences $\left.z_{n}\left(\alpha^{n}\right), \alpha^{n} \in \Sigma^{d}, n \rightarrow \infty\right\}$. It is easy to see that the above conditions about the tree $\mathcal{T}$ hold if $\mathcal{T}$ is in $W \cap \Omega$, close enough to $\operatorname{Fr} \Omega$, as in the situation of Theorem A (see Section 5).

\section{Proof of Theorem B}

STEP 1: Good backward branches and their number. Denote the natural extension of the one-sided shift $\sigma: \Sigma^{d} \rightarrow \Sigma^{d}$ preserving a Borel probability measure $\nu$, i.e. the corresponding two-sided shift, by $\left(\widetilde{\Sigma}^{d}, \widetilde{\nu}, \widetilde{\sigma}\right)$. Denote the 
projection $\widetilde{\Sigma}^{d} \rightarrow \Sigma^{d}$ mapping $\alpha$ to $\left(\alpha_{0}, \alpha_{1}, \ldots\right)$ by $\pi_{+}$. For each $\alpha \in \widetilde{\Sigma}^{d}$ denote $\pi_{+}(\alpha)$ by $\alpha^{+}$.

By Pesin theory (see [PZ, Lemma 1] for the version we apply) and by the Birkhoff Ergodic Theorem applied to $\varphi$, for every $\varepsilon>0$ we can find a set $K \subset \widetilde{\Sigma}^{d}$, constants $C, \delta>0$ and a positive integer $M$ such that $\widetilde{\nu}(K)>1-\varepsilon$ and for all $\alpha \in K$ and $n \geq 0$,

$$
\mathrm{B}(\mathrm{i})-\ln C+n\left(\int \varphi d \nu-\varepsilon / 2\right) \leq \sum_{j=0}^{n-1} \varphi\left(\sigma^{j}\left(\alpha^{+}\right)\right) \leq \ln C+n\left(\int \varphi d \nu+\varepsilon / 2\right) .
$$

$\mathrm{B}(\mathrm{ii}) b_{M}\left(\alpha^{+}\right) \subset B\left(z_{\infty}\left(\alpha^{+}\right), \delta / 3\right)$.

$\mathrm{B}(\mathrm{iii})$ There exist univalent branches $f_{\alpha}^{-n}$ of $f^{-n}$ on $B\left(z_{\infty}\left(\alpha^{+}\right), \delta\right)$ for all $n=1,2, \ldots$ mapping $z_{\infty}\left(\alpha^{+}\right)$to $z_{\infty}\left(\widetilde{\sigma}^{-n}(\alpha)^{+}\right)$.

In the notation accompanying property (1) these branches are the continuations along $b\left(\alpha^{+}\right)$of $f_{\left(\alpha_{-n}, \ldots, \alpha_{-1}\right)}^{-n}$, i.e. the branches $f_{\left(\alpha_{-n}, \ldots, \alpha_{-1}\right), \alpha^{+}}^{-n}$.

Moreover

$$
\begin{aligned}
\mathrm{B}(\mathrm{iv}) C^{-1} \exp n\left(\chi_{\mu}(f)-\varepsilon / 2\right) & \leq\left|\left(f^{n}\right)^{\prime}\left(z_{\infty}\left(\widetilde{\sigma}^{-n}(\alpha)^{+}\right)\right)\right| \\
& \leq C \exp n\left(\chi_{\mu}(f)+\varepsilon / 2\right) . \\
\mathrm{B}(\mathrm{v})\left|\left(f_{\alpha}^{-n}\right)^{\prime}(x)\right| /\left|\left(f_{\alpha}^{-n}\right)^{\prime}(y)\right| & <C \text { for all } x, y \in B\left(z_{\infty}\left(\alpha^{+}\right), \delta\right) .
\end{aligned}
$$

For $-\infty \leq r \leq s \leq \infty$ and $\alpha \in \widetilde{\Sigma}^{d}$ or $\alpha \in \Sigma_{r, s}=\{1, \ldots, d\}^{\{r, r+1, \ldots, s\}}$, we denote by $C_{r, s}(\alpha)$ the cylinder $\left\{w \in \widetilde{\Sigma}^{d}: w_{j}=\alpha_{j}\right.$ for all $\left.j: r \leq j \leq s\right\}$. The projection $\widetilde{\Sigma}^{d} \ni\left(\ldots, \alpha_{j}, \ldots\right) \mapsto\left(\alpha_{r}, \ldots, \alpha_{s}\right) \in \Sigma_{r, s}$ will be denoted by $\pi_{r, s}$. Note that $C_{r, s}(\alpha)=\pi_{r, s}^{-1} \pi_{r, s}(\alpha)$.

Choose an arbitrary cylinder $C_{M}:=C_{0, M}(\beta)$, for a fixed sequence $\beta=$ $\left(\beta_{0}, \ldots, \beta_{M}\right) \in \Sigma_{M}:=\Sigma_{0, M}$, such that $\widetilde{\nu}\left(C_{M} \cap K\right) \geq \widetilde{\nu}\left(C_{M}\right) / 2$, which is possible provided $\varepsilon \leq 1 / 2$.

Denote $C_{M} \cap K$ by $K^{\prime}$. For all $n \geq 0$ consider $K_{n}:=\widetilde{\sigma}^{-n}\left(K^{\prime}\right)$. By the invariance of $\widetilde{\nu}$ we have $\widetilde{\nu}\left(K_{n}\right) \geq \widetilde{\nu}\left(C_{M}\right) / 2=: \xi$.

By the Birkhoff Ergodic Theorem there exists $N \geq 0$ such that

$$
\nu\left(\left\{\alpha \in K_{n}: \exists i: 0 \leq i \leq N, \widetilde{\sigma}^{-i}(\alpha) \in K^{\prime}\right\} \geq \xi / 2 .\right.
$$

Therefore for every $n \geq 0$ there exists $N^{\prime}$ with $0 \leq N^{\prime} \leq N$ such that, setting $n^{\prime}:=n+N^{\prime}$, for $A\left(n^{\prime}\right):=\left\{\alpha \in K^{\prime}: \widetilde{\sigma}^{-n^{\prime}}(\alpha) \in K^{\prime}\right\}$ we have

$$
\widetilde{\nu}\left(A\left(n^{\prime}\right)\right) \geq \xi / 2 N \text {. }
$$

For every $\alpha \in A\left(n^{\prime}\right)$ we obtain $b_{M}\left(\widetilde{\sigma}^{-n^{\prime}}(\alpha)^{+}\right) \subset B\left(z_{M}\left(\alpha^{+}\right), \delta / 3\right)$. Indeed, for $\alpha^{\prime}=\sigma^{-n^{\prime}}(\alpha)$ we have $\pi_{0, M}\left(\alpha^{\prime}\right)=\beta$, as we have landed with $\alpha^{\prime}$ in $C_{M}$. The length of $b_{M}\left(\alpha^{\prime+}\right)$ is at most $\delta / 3$ as $\alpha^{\prime} \in K$.

Hence

$$
f_{\alpha}^{-n^{\prime}}\left(\operatorname{cl}\left(B\left(z_{M}(\beta), 2 \delta / 3\right)\right)\right) \subset B\left(z_{M}(\beta), 2 \delta / 3\right)
$$


for all $n$ large enough, more precisely for $n$ such that

$$
\left|\left(f_{\alpha}^{-n^{\prime}}\right)^{\prime}(x)\right|<1 / 2 \quad \text { for all } x \in B\left(z_{M}(\beta), 2 \delta / 3\right) .
$$

By B(ii)-B(iv) this holds for $n \geq(2 \ln C+\ln 2) /\left(\chi_{\mu}(f)-\varepsilon\right)$.

Claim. The branches $f_{\alpha}^{-n^{\prime}}$ on $B\left(z_{\infty}\left(\alpha^{+}\right), \delta\right)$ depend only on $\pi_{-n^{\prime}, M}(\alpha)$, more precisely on $\pi_{-n^{\prime},-1}(\alpha)$ as $\pi_{0, M}(\alpha)=\beta$ has been fixed, on the common domain $B:=B\left(z_{M}(\beta), 2 \delta / 3\right)$.

This is so since if two $\alpha^{\prime}$ 's in $A\left(n^{\prime}\right)$, say $\alpha$ and $\alpha^{\prime}$, have the same block $\left(\alpha_{-n^{\prime}}, \ldots, \alpha_{-1}\right)$, then the branches $f_{\alpha}^{-n^{\prime}}$ and $f_{\alpha^{\prime}}^{-n^{\prime}}$ are continuations of the same branch at $z$ along curves coinciding till $z_{M}(\beta)$ and next contained in the common domain $B$ (see Figure 1).

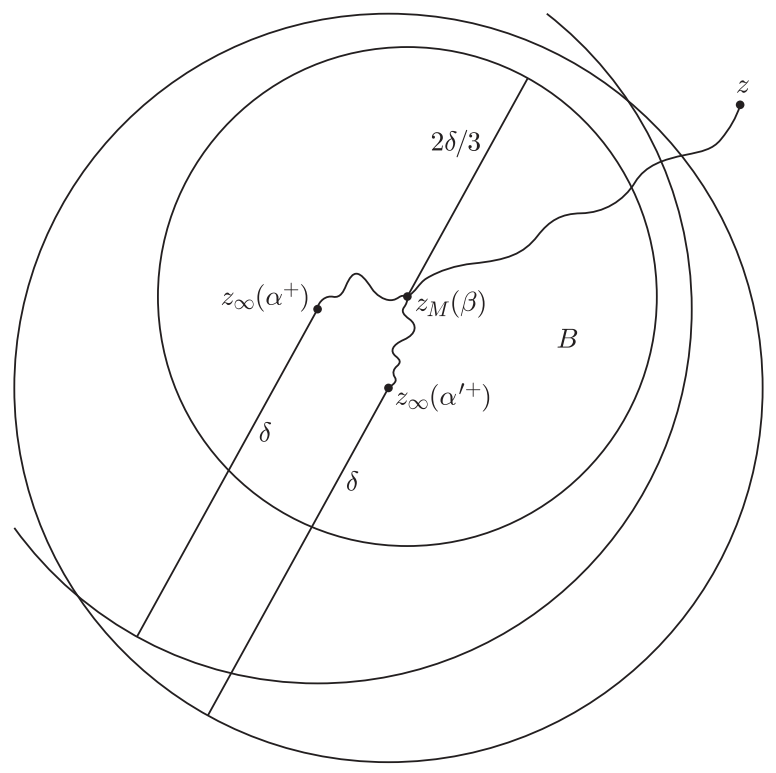

Fig. 1

We shall not use this claim directly, but we put it to help the reader understand the proof and to simplify notation later on.

By (2) we have $\widetilde{\nu}\left(\pi_{-n^{\prime}, M}^{-1} \pi_{-n^{\prime}, M}\left(A\left(n^{\prime}\right)\right)\right) \geq \xi / 2 N$. By the ShannonMcMillan-Breiman theorem for every $\eta>0$ and all integers $k$ large enough,

$$
\nu\left(\bigcup\left\{C_{0, k}(w): w \in \Sigma_{0, k}, \nu\left(C_{0, k}(w)\right) \leq \exp k\left(\mathrm{~h}_{\nu}(\sigma)+\varepsilon / 3\right)\right\}\right) \geq 1-\eta .
$$

Setting $\eta=\xi / 4 N$ and $k=M+n^{\prime}$ we get $\left.\widetilde{\nu}\left(\bigcup\left\{C_{-n^{\prime}, M}(w): w \in A\left(n^{\prime}\right)\right), \widetilde{\nu}\left(C_{-n^{\prime}, M}(w)\right) \leq \exp \left(n^{\prime}+M\right)\left(\mathrm{h}_{\nu}(\sigma)+\varepsilon / 3\right)\right\}\right)$ $\geq \xi / 2 N-\xi / 4 N=\xi / 2 N$. 
Therefore for $n$ large enough, the number of "good backward trajectories" of length $n^{\prime}$ can be estimated as follows:

$$
\#\left(\pi_{-n^{\prime},-1}\left(A\left(n^{\prime}\right)\right)\right) \geq \exp n^{\prime}\left(\mathrm{h}_{\nu}(\sigma)-\varepsilon / 2\right) .
$$

STEP 2: The sets $X, Y$ and IFS. Now define $Y^{\prime} \subset \Sigma^{d}$ as the set of onesided sequences which are concatenations of blocks $v^{k}$ belonging to $G_{n^{\prime}}:=$ $\pi_{0, n^{\prime}-1} \widetilde{\sigma}^{-n^{\prime}}\left(A\left(n^{\prime}\right)\right)$, that is,

$$
Y^{\prime}=\left\{\alpha=v^{0} v^{1} \ldots \in \Sigma^{d}: v^{k}=\pi_{0, n^{\prime}-1} \sigma^{k n^{\prime}}(\alpha) \in G_{n^{\prime}} \forall k=0,1, \ldots\right\},
$$

and set

$$
X^{\prime}=z_{\infty}\left(Y^{\prime}\right)
$$

Finally, define

$$
\begin{aligned}
& Y=\bigcup\left\{\sigma^{j}\left(Y^{\prime}\right): j=0, \ldots, n^{\prime}-1\right\}, \\
& X=\bigcup\left\{f^{j}\left(X^{\prime}\right): j=0, \ldots, n^{\prime}-1\right\}=z_{\infty}(Y) .
\end{aligned}
$$

For each $\alpha \in \Sigma^{d}$ and $r \leq s$ denote by $b_{r, s}$ the part of the branch $b(\alpha)$ starting from $z_{r-1}(\alpha)$ and ending at $z_{s}(\alpha)$.

Now, to put it briefly, by (3) and (4) for every $\alpha \in Y^{\prime}$ the length of $b_{k n^{\prime},(k+1) n^{\prime}-1}(\alpha)$ is less than $C 2^{-k}$ for a constant $C>0$. Hence $z_{n}(\alpha) \rightarrow$ $z_{\infty}(\alpha)$ uniformly (even exponentially fast), which proves (ii) on $Y^{\prime}$, hence on $Y$ by the uniform continuity of $f$. By (3) and (4), $X^{\prime}$, and hence $X$, are expanding repellers for $f^{n^{\prime}}$ and $f$ respectively.

Let us now be more precise. Let $\alpha \in Y^{\prime}$ be a concatenation of $v^{k}=$ $\pi_{0, n^{\prime}-1} \widetilde{\sigma}^{-n^{\prime}}\left(w^{k}\right)$, for $w^{k} \in A\left(n^{\prime}\right)$, for $k=0,1, \ldots$ We want to analyse $b(\alpha)$. Note that by $(1)$,

$$
b_{(k-1) n^{\prime}, k n^{\prime}-1}(\alpha)=f_{v^{0}, v^{1} v^{2} \ldots v^{k}}^{-n^{\prime}}\left(\ldots\left(f_{v^{k-1}, v^{k}}^{-n^{\prime}}\left(b_{0, n^{\prime}-1}\left(v^{k}\right)\right)\right)\right) .
$$

Assume that $n^{\prime}>M$. Then all $b(\alpha)$ for $\alpha=v^{0} v^{1} \ldots \in Y^{\prime}$ pass through $z_{M}(\beta)$ since $v^{0} \in G_{n^{\prime}}$ implies that $b_{0, n^{\prime}-1}(\alpha)$ depending only on $v^{0}$ passes through $z_{M}(\beta)$. (There is no reason for $\alpha$ to belong to $A\left(n^{\prime}\right)$, which would imply passing through $z_{M}(\beta)$ by definition, as in the Claim. So for the first time in the proof we need to use $n^{\prime}>M$.)

Now we apply induction on $k$. Suppose that for every $\alpha \in \Sigma_{0, k n^{\prime}-1}$ which is a concatenation $v^{0} v^{1} \ldots v^{k-1}$ of blocks $v^{j}$ in $G_{n^{\prime}}$ we have $b_{M+1, k n^{\prime}-1}(\alpha)$ $\subset B$ (see Figure 2)). Take an arbitrary $v \in G_{n^{\prime}}$ which is the truncation of $w \in A\left(n^{\prime}\right)$, more precisely $v=\pi_{0, n^{\prime}-1} \widetilde{\sigma}^{-n^{\prime}}(w)$. Then $f_{v, \alpha}^{-n^{\prime}}$ and $f_{w}^{-n^{\prime}}$ coincide on $B$, in particular on $b_{M, k n^{\prime}-1}(\alpha)$, since also $b_{M}\left(w^{+}\right)$is contained in $B$, as $w \in K$, yielding a path in $\mathcal{T}$ joining $z_{k n^{\prime}-1}(\alpha)$ to $z_{\infty}\left(w^{+}\right)$and entirely contained in $B$ (compare the proof of Claim). Hence, by (3) applied to $f_{w}^{-n^{\prime}}$ we get $b_{M+1,(k+1) n^{\prime}-1}(v \alpha) \subset B$, which finishes the induction. 


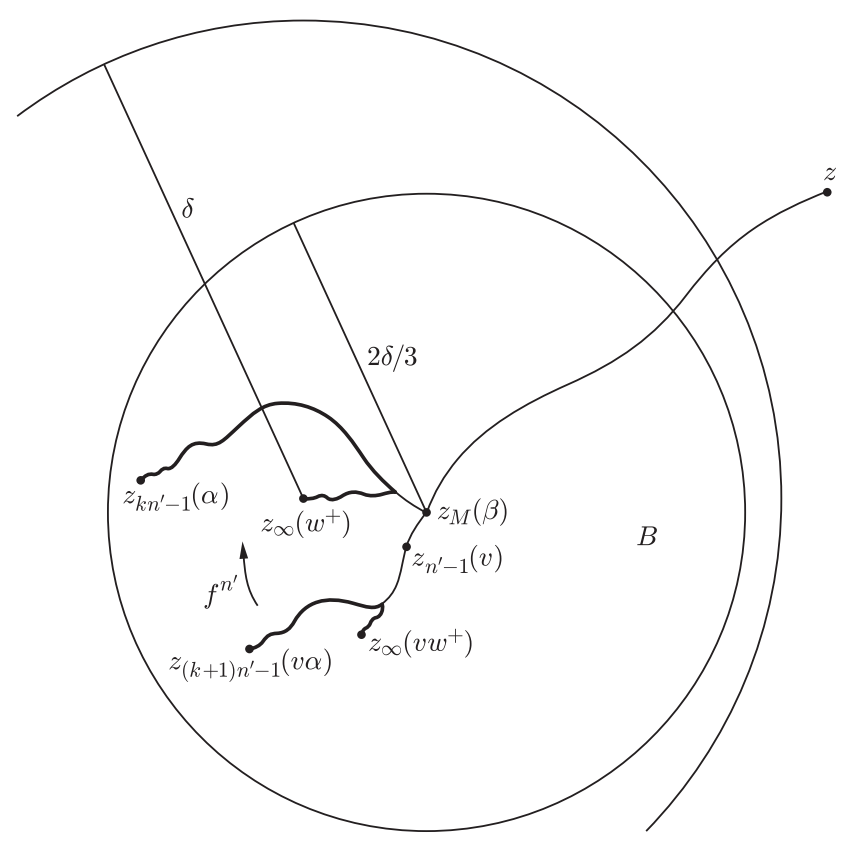

Fig. 2

Therefore in (6) we can replace $f_{v^{j}, v^{j+1} v^{j+2} \ldots v^{k}}^{-n^{\prime}}$ by $f_{w^{j}}^{-n^{\prime}}$ for all $j=$ $0,1, \ldots, k-1$, in particular these branches of $f^{-n^{\prime}}$ act on branches of the tree $\mathcal{T}$ in the common domain $B$ (except $\left.b_{0, n^{\prime}-1}\left(v^{k}\right)\right)$.

One can view the family of branches $F_{v}:=f_{v}^{-n^{\prime}}$ for $v \in G_{n^{\prime}}$ as an iterated function system (IFS) on $B$. It satisfies the so-called Strong Open Set Condition, i.e. all $F_{v}(B)$ have pairwise disjoint closures. The Claim allows us to write $v$ in place of $w$, where $v$ is the truncation of $w$. These branches also act on (extend to) $b_{0, M}(\beta)$, the line in the tree joining $z$ to $z_{M}(v)$ which need not be contained in $B$. So $F_{v}$ need not contract it. But further iteration contracts them exponentially since $F_{v}\left(b_{0, M}(\beta)\right)$ lies already in $B$.

The limit set is contained in $\mathrm{cl} z_{\infty}\left(\Sigma^{d}\right)$, since the $F_{v}$ preserve the tree $\mathcal{T}$.

SteP 3: Proving properties (i)-(ix) in Theorem B. To prove (i) consider an arbitrary $\alpha=v^{0} v^{1} \ldots \in Y^{\prime}$ for $v^{k}=\pi_{0, n^{\prime}-1} \widetilde{\sigma}^{-n^{\prime}}\left(w^{k}\right)$, where $w^{k} \in A\left(n^{\prime}\right)$. Then, for each $k=1,2, \ldots$,

$$
\sum_{j=0}^{k n^{\prime}-1} \varphi\left(\sigma^{j}\left(\alpha^{+}\right)\right)-\sum_{i=0}^{k-1} \sum_{j=0}^{n^{\prime}-1} \varphi\left(\sigma^{j}\left(\left(\widetilde{\sigma}^{-n^{\prime}}\left(w^{i}\right)\right)^{+}\right)\right) \leq k n^{\prime} \varepsilon / 2
$$

for $n$ large enough. This follows from the continuity of $\varphi$ since $\sigma^{i n^{\prime}+j}(\alpha)$ and $\sigma^{j}\left(\left(\widetilde{\sigma}^{-n^{\prime}}\left(w^{i}\right)\right)^{+}\right)$are very close to each other for all $i$ and $0 \leq j \ll n^{\prime}$. This is so because both one-sided sequences have the same beginning of length 
$n^{\prime}-j$. Now (i) follows from the estimate B(i) on $\sum_{j=0}^{n^{\prime}-1} \varphi\left(\sigma^{j}\left(\left(\widetilde{\sigma}^{n^{\prime}}\left(w^{i}\right)\right)^{+}\right)\right.$. Passing from $Y^{\prime}$ to $Y$ changes only the constant $C$ in (i).

These considerations also prove (vi). Indeed, in the case of $\psi$ one ensures the property of $K$ analogous to B(i), namely

$$
\begin{aligned}
\mathrm{B}(\mathrm{vi})-\ln C+n\left(\int \psi d \mu-\varepsilon / 2\right) & \leq \sum_{j=0}^{n-1} \psi\left(f^{j}\left(z_{\infty}\left(\alpha^{+}\right)\right)\right) \\
& \leq \ln C+n\left(\int \psi d \mu+\varepsilon / 2\right),
\end{aligned}
$$

following from the $\nu$-integrability of $\psi \circ z_{\infty}$ and the Birkhoff Ergodic Theorem. Use also the property analogous to (7), for $\psi$ and $f$ in place of $\varphi$ and $\sigma$, which follows from the continuity of $\psi$ and the fact that the preimages of points in $B$ under the same branch $f^{j} f_{v^{i}}^{-n^{\prime}}$ of $f^{-\left(n^{\prime}-j\right)}$ are very close to each other for $0 \leq j \ll n^{\prime}$.

The uniform (exponential) convergence in (ii) has already been proven. The injectivity and the property of $X^{\prime}$ of being a Cantor set follow from the Strong Open Set Condition of the IFS $\left\{F_{v}\right\}$. This implies that $z_{\infty}$ is finite-to-one on $Y$ and $X$ is also a Cantor set.

By (5) and (i) and by the definition of pressure,

$$
\mathrm{P}\left(\left.\sigma^{n^{\prime}}\right|_{Y^{\prime}}, \sum_{j=0}^{n^{\prime}-1} \varphi \circ \sigma^{j}\right) \geq \mathrm{h}_{\nu}\left(\sigma^{n^{\prime}}\right)+n^{\prime}\left(\int \varphi d \nu-\varepsilon\right),
$$

hence easily $\mathrm{P}\left(\left.\sigma\right|_{Y}, \varphi\right) \geq \mathrm{h}_{\nu}(f)+\int_{Y} \varphi d \nu-\varepsilon$, proving (vii) for $\mathrm{P}\left(\left.\sigma\right|_{Y}, \varphi\right)$. The argument for $\mathrm{P}\left(\left.f\right|_{X}, \psi\right)$ is similar, using (vi) for $\psi$.

Note that one cannot pull back to $\Sigma^{d}$ to refer to (vii) for $\mathrm{P}\left(\sigma, \psi \circ z_{\infty}\right)$ on $Y$ since $\psi \circ z_{\infty}$ need not be continuous on $\partial \mathbb{D}$, even not defined, so we might not have (7).

By $[\mathrm{M}]$, or [P1, Sec. 3] where further references are provided, we have $\operatorname{HD}(\mu)=\mathrm{h}_{\mu}(f) / \chi_{\mu}(f)$. Consider an arbitrary $\varepsilon^{\prime}>0$ and set $t^{\prime}:=\operatorname{HD}(\mu)-\varepsilon^{\prime}$. Then $t^{\prime}=\mathrm{h}_{\mu}(f) / \chi_{\mu}(f)-\varepsilon^{\prime}$. By (iii) and (5),

$$
\begin{aligned}
\mathrm{P}\left(\left.f\right|_{X},-t^{\prime} \ln \left|f^{\prime}\right|_{X} \mid\right) & \geq \mathrm{h}_{\text {top }}\left(\left.f\right|_{X}\right)-t^{\prime}\left(\chi_{\mu}(f)+\varepsilon\right) \\
& \geq \mathrm{h}_{\mu}(f)-\varepsilon-\left(\mathrm{h}_{\mu}(f) / \chi_{\mu}(f)-\varepsilon^{\prime}\right)\left(\chi_{\mu}(f)+\varepsilon\right) \\
& \geq-\varepsilon-\varepsilon \mathrm{h}_{\mu}(f) / \chi_{\mu}(f)+\varepsilon^{\prime} \chi_{\mu}(f)+\varepsilon \varepsilon^{\prime},
\end{aligned}
$$

which is positive if

$$
\varepsilon^{\prime}>\frac{\varepsilon\left(1+\mathrm{h}_{\mu}(f)\right) / \chi_{\mu}(f)}{\chi_{\mu}(f)+\varepsilon} .
$$

Hence $\operatorname{HD}(X)>t^{\prime}$ as $\operatorname{HD}(X)$ is not smaller than the first zero of the pressure function $t \mapsto \mathrm{P}\left(\left.f\right|_{X},-t \ln \left|f^{\prime}\right|_{X} \mid\right.$ ), by the Bowen theorem (see for 
example [PU]). If we choose $\varepsilon$ small we obtain $\varepsilon^{\prime}$ small, hence $\operatorname{HD}(X)$ arbitrarily close to $\operatorname{HD}(\mu)$, which proves (iv).

We prove (ix) similarly.

To prove (v) consider the cylinder $C_{M}=C_{0, M}(\beta)$ for $\beta$ being the truncation of a sequence $\alpha$ dense in $\operatorname{supp} \nu$ and $M$ large. The proof of Theorem B is finished.

5. Conclusions. Theorem B easily implies Theorem A. One builds the tree $\mathcal{T}$ in the basin of attraction. It is only sufficient to note that the branches of the tree $R^{-1}(\mathcal{T})$ converge to $\partial \mathbb{D}$ nontangentially, so the convergence of each branch $b(\alpha)$ in $\mathcal{T}$ implies the nontangential, in particular radial, convergence of $R$ at $\lim R^{-1}(b(\alpha)) \in \partial \mathbb{D}$, with the same limit. One considers the pull-back $\varphi \circ\left(R^{-1}(z)\right)_{\infty}: \Sigma^{d} \rightarrow \mathbb{R}$, finds $Y$ in $\Sigma^{d}$, maps it by $\left(R^{-1}(z)\right)_{\infty}$ with the use of $R^{-1}(\mathcal{T})$ into $\partial \mathbb{D}$ and with the use of $\mathcal{T}$ to $X \subset \operatorname{Fr} \Omega$ as in Theorem B. The map $\widehat{R}$ is finite-to-one on $Y$ since $z_{\infty}$ is. The rate of the exponential convergence in (ii) and more precisely in (ii') in Remark 2 follows easily from (iii), (i) applied to $\varphi=\ln \left|g^{\prime}\right|$, and the chain rule $R^{\prime}(z)=\left(f^{-n}\right)^{\prime}\left(R\left(g^{n}(z)\right)\right) \cdot R^{\prime}\left(g^{n}(z)\right) \cdot\left(g^{n}\right)^{\prime}(z)$ for $z=r \zeta$, the integer $n$ such that for the first time $g^{n}(z)$ is far from $\partial \mathbb{D}$, and the appropriate branch of $f^{-n}$; for details see [P2]. See also [P3].

REMARK 3. If $\nu$ is mixing, which is the case for Gibbs $\nu$ as in Remark 1 , then one can ensure that $f$ on $X$ is topologically mixing, that is, for any open subsets $U, V$ of $X$ there exists $n_{0}$ such that $f^{n}(U) \cap V \neq \emptyset$ for all $n \geq n_{0}$.

Indeed, for $n$ large we have by mixing $\widetilde{\nu}\left(\widetilde{\sigma}^{-n}\left(C_{M}\right) \cap C_{M}\right) \sim \nu\left(C_{M}\right)^{2}$. Hence, if $\nu(K) \approx 1$, then $\widetilde{\nu}(A(n)) \geq$ const $>0$ for all $n$ large (compare (2)). We can repeat the previous construction by taking instead of one $n^{\prime}$ two different mutually prime integers.

REMARK 4. Theorem A holds in the case $\Omega$ is an immediate connected simply connected basin of attraction to a parabolic fixed point $p$, i.e. $p \in \operatorname{Fr} \Omega$ such that $f(p)=p$ and $f^{\prime}(p)$ is a root of unity.

Indeed, in this case $R^{-1} \circ f \circ R$ extends to $\overline{\mathbb{C}}$ to yield $g$ which is a Blaschke product such that $\mathbb{D}$ (and $\overline{\mathbb{C}} \backslash \mathrm{cl} \mathbb{D}$ ) is a basin of a parabolic fixed point for $g$ in $\partial \mathbb{D}$. As in the conclusion that Theorem B implies Theorem A, we consider the trees $\mathcal{T}$ and $R^{-1}(\mathcal{T})$. All the branches of $R^{-1}(\mathcal{T})$ converge (polynomially fast, but not necessarily nontangentially), and at each limit point $\zeta=\left(R^{-1}(z)\right)_{\infty}(\alpha)$ for $\alpha \in \mathcal{D}\left(z_{\infty}\right)$, in particular in $Y$, the radial limit $\widehat{R}(\zeta)$ coincides with $z_{\infty}(\alpha)$ by Lindelöf's theorem. Hence $z_{\infty}=$ $\widehat{R} \circ\left(R^{-1}(z)\right)_{\infty}$ on $Y$ and all the maps involved are finite-to-one since $z_{\infty}$ is finite-to-one on $Y$. 


\section{References}

[HK] A. Katok and B. Hasselblatt, Introduction to the Modern Theory of Dynamical Systems, with supplement by A. Katok and L. Mendoza, Cambridge Univ. Press, 1995.

[M] R. Mañé, The Hausdorff dimension of invariant probabilities of rational maps, in: Dynamical Systems (Valparaiso, 1986), Lecture Notes in Math. 1331, Springer, 1988, 86-117.

[P1] F. Przytycki, Hausdorff dimension of harmonic measure on the boundary of an attractive basin for a holomorphic map, Invent. Math. 80 (1985), 161-179.

[P2] —, Riemann map and holomorphic dynamics, ibid. 85 (1986), 439-455.

[P3] -, An improvement of J. Rivera-Letelier result on weak hyperbolicity on periodic orbits for polynomials, submitted; http://www.impan.gov.pl/ feliksp.

[PS] F. Przytycki and J. Skrzypczak, Convergence and pre-images of limit points for coding trees for iterations of holomorphic maps, Math. Ann. 290 (1991), 425-440.

[PU] F. Przytycki and M. Urbański, Fractals in the Plane, Ergodic Theory Methods, Cambridge Univ. Press, to appear; http://www.math.unt.edu/ urbanski and http://www.impan.gov.pl/ feliksp.

[PUZ] F. Przytycki, M. Urbański and A. Zdunik, Harmonic, Gibbs and Hausdorff measures for holomorphic maps, Part 1, Ann. of Math. 130 (1989), 1-40; Part 2, Studia Math. 97 (1991), 189-225.

[PZ] F. Przytycki and A. Zdunik, Density of periodic sources in the boundary of a basin of attraction for iteration of holomorphic maps: geometric coding trees technique, Fund. Math. 145 (1994), 65-77.

Institute of Mathematics

Polish Academy of Sciences

Śniadeckich 8

00-956 Warszawa, Poland

E-mail: feliksp@impan.gov.pl

Received 7 February 2005 